\title{
CHEBYSHEV APPROXIMATION WITH A NULL SPACE
}

\author{
CHARLES B. DUNHAM
}

\begin{abstract}
Chebyshev approximation involving continuous functions vanishing on a closed set $V$ is considered. The approximating families studied have the betweenness property. Examples are given of such families. A necessary and sufficient condition for uniqueness of best approximations is obtained.
\end{abstract}

Let $X$ be a compact space and $V$ be a closed subset of $X$. Let $C(V, X)$ be the space of continuous functions on $X$ which vanish on $V$. For $g \in C(V, X)$ define

$$
\|g\|=\sup \{|g(x)|: x \in X\} .
$$

Let $\mathscr{G}$ be a subset of $C(V, X)$. The Chebyshev problem is: Given $f \in$ $C(V, X)$, find $G^{*}$ in $\mathscr{G}$ to minimize $e(G)=\|f-G\|$. Such an element $G^{*}$ is called a best approximation in $\mathscr{G}$ to $f$ on $X$.

At least two cases of such a problem arise naturally, namely approximation with functions vanishing at zero and approximation with functions decaying to zero at infinity.

A seemingly more general problem is to approximate with functions which agree with $v \in C(X)$ on a closed subset $V$ of $X$. This problem, however, reduces to the previous problem if we subtract $v$ from all functions.

We consider the best approximation problem and in particular the uniqueness problem if $\mathscr{G}$ has the betweenness property [1].

Definition. A family $\mathscr{G}$ of continuous functions is said to have the betweenness property if for any two elements $G_{0}$ and $G_{1}$, there exists a $\lambda$ set $\left\{H_{\lambda}\right\}$ of elements of $\mathscr{G}$ such that $H_{0}=G_{0}, H_{1}=G_{1}$ and for all $x \in X$, $H_{\lambda}(x)$ is either a strictly monotonic continuous function of $\lambda$ or a constant, $0 \leqq \lambda \leqq 1$.

EXAMPLE. Let $\mathscr{G}$ be a linear subspace of $C(V, X)$, then $\mathscr{G}$ has the betweenness property, for a $\lambda$-set is given by $H_{\lambda}=\lambda G_{1}+(1-\lambda) G_{0}$.

EXAMPLE. Let $P$ be a linear subspace of $C(V, X)$ and $Q$ a linear subspace of $C(X)$ then $\mathscr{G}=\{p / q: p \in P, q \in Q, q>0\}$ is in $C(V, X)$ and has the betweenness property $[1,152]$.

Received by the editors January 22, 1973.

AMS (MOS) subject classifications (1970). Primary 41A50.

Key words and phrases. Chebyshev approximation, betweenness property, uniqueness.

(c) American Mathematical Society 1973 
Lemma. Let $\sigma$ be a continuous strictly monotonic mapping of the real line into the real line such that $\sigma(0)=0$.

Let $\mathscr{G} \in C(V, X)$ have the betweenness property. Then

$$
\phi(\mathscr{G})=\{\sigma(G): G \in \mathscr{G}\} \subset C(V, X)
$$

and has the betweenness property.

Proof. Let $\left\{H_{\lambda}\right\}$ be a $\lambda$-set for $G_{0}$ and $G_{1}$. Then $\left\{\sigma\left(H_{\lambda}\right)\right\}$ is a $\lambda$-set for $\sigma\left(G_{0}\right)$ and $\sigma\left(G_{1}\right)$.

Lemma. Let $\mathscr{G} \subset C(W, X)$ have the betweenness property and $s \in$ $C(V, X)$. Then the set $s \mathscr{G}$ (consisting of products of $s$ and elements of $\mathscr{G})$ is in $C(W \cup V, X)$ and has the betweenness property.

The previous theory obtained for betweenness [1] gives a characterization of best approximations and an error-determining set on which best approximations agree. We must, however, develop a new theory for uniqueness.

Definition. $\mathscr{G} \subset C(V, X)$ has zero-sign compatibility with null space $V$ if for any two distinct elements $G$ and $H$, any closed subset $Z$ of the zeros of $G-H$ which contains no points of $V$ and for any $s \in C(V, X)$ taking values -1 or +1 on $Z$, there exists $F \in \mathscr{G}$ such that

$$
\operatorname{sgn}(F(X)-G(x))=s(x), \quad x \in Z .
$$

THEOREM. Let $\mathscr{G} \subset C(V, X)$ have the betweenness property. A necessary and sufficient condition that for every $f \in C(V, X)$ a best approximation is unique is that $\mathscr{G}$ have zero-sign compatibility with null space $V$.

The proof is the same as the proof of the corresponding result in [1].

The case where $\mathscr{G}$ is a finite-dimensional linear family is of particular interest. It can be shown using the above theorem that a necessary and sufficient condition for uniqueness is that $\mathscr{G}$ be a Haar subspace on $X \sim V$. Independent proofs of necessity and sufficiency are given in [3], [2], respectively.

\section{REFERENCES}

1. C. B. Dunham, Chebyshev approximation by families with the betweeness property, Trans. Amer. Math. Soc. 136 (1969), 151-157. MR 38 \#4880.

2. - Linear Chebyshev approximation, Aequationes Math. (to appear).

3. - - Families satisfying the Haar condition, J. Approximation Theory (to appear).

Department of Computer Science, University of Western Ontario, London, Ontario, Canada 